Research Paper

\title{
Receptor for activated $C$ kinase 1 in rats with ischemia-reperfusion injury: intravenous versus inhalation anaesthetic agents
}

\author{
Eun-Hye Seo ${ }^{1}$, Ga-Yun Song², Ji Hyeon Namgung², Chung-Sik $\mathrm{Oh}^{3}$, Seung Hyun Lee²,3, Seong-Hyop \\ $\mathrm{Kim}^{3,4,5 凶}$ \\ 1. BK21 Plus, Department of Cellular and Molecular Medicine, Konkuk University School of Medicine, Seoul, Korea \\ 2. Department of Microbiology, Konkuk University School of Medicine, Seoul, Korea \\ 3. Department of Medicine, Institute of Biomedical Science and Technology, Konkuk University School of Medicine, Seoul, Korea. \\ 4. Department of Anesthesiology and Pain medicine, Konkuk University Medical Center, Konkuk University School of Medicine, Seoul, Korea. \\ 5. Department of Infection and Immunology, Konkuk University School of Medicine, Seoul, Korea.
}

$\square$ Corresponding author: Seong-Hyop Kim, M.D., Ph.D. Address: Department of Anesthesiology and Pain medicine, Konkuk University Medical Center, Konkuk University School of Medicine, 120-1 Neudong-ro (Hwayang-dong), Gwangjin-gu, Seoul 05030, Korea. Tel: +82-2-2030-5454, Fax: +82-2-2030-5449, E-mail: yshkim75@daum.net

(c) Ivyspring International Publisher. This is an open access article distributed under the terms of the Creative Commons Attribution (CC BY-NC) license (https://creativecommons.org/licenses/by-nc/4.0/). See http://ivyspring.com/terms for full terms and conditions.

Received: 2017.08.29; Accepted: 2018.01.12; Published: 2018.02.12

\begin{abstract}
Background: The study examined the difference in the expression of the receptor for activated $C$ kinase 1 (RACK1) between anaesthesia with propofol and isoflurane in rats with myocardial ischemia-reperfusion injury (IRI).

Methods: Male Sprague-Dawley rats were studied. Anaesthesia was induced with xylazine $20 \mu \mathrm{g} / \mathrm{g}$ by intraperitoneal injection and maintained with propofol or isoflurane. Myocardial IRI was induced by ligating the left anterior descending artery for 1 hour. Reactive oxygen species (ROS), cardiomyocyte apoptosis, the expression of RACK1 and toll-like receptor 4 (TLR4), and the heart injury score were compared between the two groups.

Results: Cardiomyocyte apoptosis with ROS was significantly lower in the propofol group than in the isoflurane group. The propofol group had significantly higher RACK1 expression and lower TLR4 expression, compared with the isoflurane group (RACK1, $1970.50 \pm 120.50$ vs. $1350.20 \pm$ $250.30, p<0.05$; TLR4, $980.50 \pm 110.75$ vs. $1275.50 \pm 75.35, p<0.05)$. However, the heart injury scores in the two groups did not differ significantly ( $3.56 \pm 0.29$ vs. $4.33 \pm 0.23$ in the propofol and isoflurane groups, respectively, $p=0.33$ ).

Conclusion: There were significant differences in inflammation and apoptosis, including the expression of RACK1 and TLR4, after myocardial IRI between the propofol and isoflurane groups. However, both groups had similar heart injury scores.
\end{abstract}

Key words: Receptor for activated C kinase 1, Propofol, Isoflurane, Myocardial ischemia-reperfusion injury

\section{Introduction}

Ischemia-reperfusion injury (IRI) is defined as the tissue damage caused when the blood supply returns to a tissue after ischemia $[1,2]$. The restoration of circulation after the absence of oxygen and nutrients from blood during the ischemic period paradoxically induces pathophysiological processes that include the accumulation of reactive oxygen species (ROS) and release of inflammatory cytokines, resulting in cell apoptosis [3-6]. Consequently, myocardial IRI produces cardiac injury, cardiac contractile dysfunction, and increased myocardial infarct size.

Recently, the receptor for activated C kinase 1 (RACK1) was reported to perform an anti-apoptosis 
role in cardiomyocytes after myocardial IRI [7-9]. It mediates protein kinase $\mathrm{C}$ (PKC) signal transduction in cardiomyocytes and its over-expression results in the suppression of apoptosis [10,11].

Numerous studies have investigated the role of anaesthetic agents in myocardial IRI [13, 14]. However, the association between RACK1 and myocardial IRI with specific anaesthetic agents has not been investigated.

We hypothesised that a difference in RACK1 expression between intravenous and inhalation anaesthetic agents would exist in myocardial IRI. Therefore, this study examined the difference in the expression of RACK1 between propofol and isoflurane anaesthesia in the rat with myocardial IRI.

\section{Materials and Methods}

\section{Experiment design}

The study was approved by the Konkuk University Institutional Animal Care and Use Committee (KU16071-1). Male Sprague-Dawley (SD) rats 6-8 weeks in age and weighing $\sim 200 \mathrm{~g}$ were purchased from Orient Bio (Seongnam, Korea). The animal experiments followed the National Institutes of Health guidelines for care, and all animals were handled for 7 days before starting the experiments. Anaesthesia was induced by intraperitoneal injection of xylazine $20 \mu \mathrm{g} / \mathrm{g}$ (Rompun ${ }^{\circledR}$, Bayer Korea, Korea). Anaesthesia was assessed by pinching the hind foot. Tracheal intubation was performed at surgery. A heating pad was placed on the surgery platform to maintain the rat body temperature during surgery. With the rat in a supine position, it was fastened to the platform with tape, and its tongue was pulled out with forceps. Intubation was performed using a 1.77-inch-long 16 gauge catheter (BD, USA) inserted through the larynx to the bronchus. The correct position of the intubation catheter was confirmed by checking for symmetric chest expansion. A ventilator (Harvard Apparatus, Holliston, MA, USA) was connected to the intubation catheter and set to 1) a fraction of inspired oxygen $\left(\mathrm{FiO}_{2}\right)$ of $\left.0.5,2\right)$ an inspiratory flow rate of $170 \mathrm{~mL} /$ minute, 3) a tidal volume of $6 \mathrm{~mL} / \mathrm{kg}$, and 4) a respiratory rate of 80 breaths/minute [15]. The rats were randomly divided into propofol and isoflurane groups, in which anaesthesia was maintained with propofol or isoflurane, respectively. For propofol (Dongkook Pharmaceutical, Korea), a $10 \mathrm{mg} / \mathrm{mL}$ solution in a 50 $\mathrm{ml}$ syringe was continuously administered using an infusion pump (Masterflex L/S peristaltic pump with Masterflex L/S easy load pump head and L/S tubing, Cole-Parmer Instrument, Vernon Hills, IL, USA) at 5 $\mu \mathrm{L} / \mathrm{g} /$ hour $(50 \mu \mathrm{g} / \mathrm{g} /$ hour) via a tail vein. The isoflurane (JW Pharmaceutical, Korea) was administered using a vaporiser at $3 \%$ volume via the intubation catheter.

\section{IRI on heart}

One hour after inducing anaesthesia, the rat was carefully turned so that it was lying on its right side and facing its left side. The chest was shaved and alcohol and povidone (Firson, Korea) were applied to prevent contamination of the surgical field. A left thoracotomy was performed between the $3^{\text {rd }}$ and $4^{\text {th }}$ ribs, and the skin and muscle were dissected carefully. A chest retractor was placed in the incision and the pericardium was picked up gently with forceps. The left anterior descending artery (LAD) was identified between the pulmonary artery and left auricle and ligated with 4-0 silk (Covidien, Dublin, Ireland). The cardiac ischemia was evaluated as successful when the colour of the territory of the LAD changed to light red after ligation. The retractor was removed and the surgical field was covered with wet gauze for 1 hour. Then, the ligature around the LAD was released to allow reperfusion. Reperfusion was confirmed when the colour in the territory of the LAD changed to dark red. The surgical field was covered with wet gauze for 4 hours under anaesthesia and the rats were sacrificed. After laparotomy, a $3 \mathrm{~mL}$ blood sample in an ethylenediaminetetraacetic acid (EDTA) tube was obtained from the hepatic vein to measure the ROS level. The heart was extracted to obtain cardiomyocytes and for histology and immunohistochemistry.

\section{Flow Cytometry}

The blood in the EDTA tube was transferred to a conical tube and diluted to $10 \mathrm{~mL}$ with phosphate-buffered saline (PBS; Gibco, USA). Peripheral blood mononuclear cells (PBMCs) were isolated from the blood using density-gradient centrifugation over a Biocoll gradient solution (Biochrom, German). The PBMCs were washed with fluorescence activated cell sorter (FACS) buffer [1\% bovine serum albumin (BSA) and $0.01 \% \mathrm{NaN}_{3}$ in PBS]. After washing the PBMCs, they were stained with 2',7'-dichlorofluorescein diacetate (H2DCFDA, Life Technologies, USA), changing from non-fluorescent into fluorescent on oxidation, to detect intracellular ROS. The staining was performed for 30 minutes in the dark at room temperature and the sample was analysed on a flow cytometer. The data were analysed using FlowJo software (Tree Star, USA).

\section{Cardiomyocytes}

To confirm apoptosis and the expression of RACK1, cardiomyocytes were cultured. Heart tissue was digested with Hanks balanced salt solution 
(HBSS; Gibco, USA) and incubated in a conical tube with an enzyme solution in a $37^{\circ} \mathrm{C}$ water bath for 30 minutes. The enzyme solution consisted of $0.1 \%$ collagenase type 2 (Sigma-Aldrich, USA) and 0.5\% trypsin (Gibco, USA) in HBSS. After incubation, the supernatant was collected. The cells from the supernatant were harvested using a centrifuge at 700 $g$ for 10 minutes and suspended in Dulbecco's modified Eagle's medium F-12-1 (DMEM F-12-1, Gibco, USA) containing $5 \%$ foetal bovine serum (FBS; Thermo Fisher Scientific, USA), heat-inactivated horse serum (HS; Vector, USA), and $1 \%$ penicillin-streptomycin (Gibco, USA). After centrifugation, the pellet was plated on a $100 \mathrm{~mm}$ dish and incubated for 1 hour at $37^{\circ} \mathrm{C}$ in a carbon dioxide incubator. After incubation, the supernatant in the dish was harvested and seeded on another dish with DMEM F-12-1. After cardiomyocytes were obtained via cell culture, as described above, cardiomyocyte apoptosis was evaluated using flow cytometry. The cultured cardiomyocytes were stained with tetramethylrhodamine, methyl ester, perchlorate (TMRM; Thermo Fisher scientific, USA), and annexin- $\mathrm{V}$ (BioLegend, USA). The staining was performed for 30 minutes in the dark at room temperature and the sample was analysed using flow cytometry. The data were analysed with FlowJo software.

\section{Expression of RACK1}

To confirm the expression of RACK1, immunohistochemistry and Western blotting were performed.

Immunohistochemical staining for RACK1 (Abcam, USA) and toll-like receptor 4 (TLR4, Abcam, USA) was performed using 4- $\mu \mathrm{m}$ tissue slices, which were deparaffinised and prepared for epitope retrieval using citrate buffer at $\mathrm{pH} 6$. The slices were incubated in blocking solution for 1 hour. After incubation, they were reacted with primary antibody against mouse-RACK1 (Abcam, USA) overnight at $4^{\circ} \mathrm{C}$. Then, they were incubated for 1 hour with diluted biotinylated secondary antibody. After reacting with the secondary antibody, avidin-biotin complex reagent (Vector, USA) was applied for 1 hour at room temperature and followed by 3,30-diaminobenzidine reagent (Vector, USA). The slices were stained with haematoxylin as a counter stain, rehydrated, and cover-slipped using mounting medium (Vector, USA).

For immunohistochemical staining for TLR4, antibody against TLR4 rabbit polyclonal antibody (Abcam, USA) diluted 1:100 was used as the primary antibody. Photographs were taken using a microscope (Nikon, Japan). The RACK1 and TLR4 intensities were quantified using NIH Image J software.

For Western blotting, the cultured cardiomyocytes were homogenised in lysis buffer (150 mM NaCl, $1.0 \%$ nonyl phenoxypolyethoxylethanol-40, and $50 \mathrm{mM}$ Tris $\mathrm{HCl}$; ElpisBio, Korea) containing protease inhibitor (Sigma-Aldrich, USA) and clarified by centrifugation at $13,000 \mathrm{rpm}$ for 15 minutes at $4^{\circ} \mathrm{C}$ to collect the supernatant. The proteins in the supernatant were resolved by $7.5 \%$ sodium dodecyl sulphate-polyacrylamide gel electrophoresis (SDS-PAGE, Bio-Rad) and separated. They were transferred to a polyvinylidene difluoride membrane (PVDF; Millipore, USA) using transfer apparatus for 2 hours at $300 \mathrm{~mA}$. The membrane was blocked with $5 \%$ BSA for 2 hours at room temperature and incubated with primary antibodies against mouse-RACK1 (Santa Cruz Biotechnology, USA at $4^{\circ} \mathrm{C}$ overnight. The membranes were incubated with horseradish peroxidase-conjugated secondary antibodies (Abcam, USA). The protein was detected using an LAS-3000 imaging system (Fujifilm, Japan). For re-probing, the blots were stripped with western Blot Stripping Buffer (Thermo Scientific, USA). The blot was then re-blocked and re-probed with rabbit-cleaved caspase-3 (Santa Cruz Biotechnology, USA), mouse-Bcl-2 (Santa Cruz Biotechnology, USA) and rabbit-glyceraldehyde 3-phosphate dehydrogenase (GAPDH, Sigma-Aldrich, USA) antibody detected with ECL substrate and LAS-3000 imaging system (Fujifilm, Japan).

\section{Histological analysis of heart}

Heart tissue was fixed overnight at room temperature in $4 \%$ paraformaldehyde solution (PFA; BIOSESANG, Korea). After washing the heart tissue, it was embedded in paraffin blocks. The tissues were cut in $4 \mu \mathrm{m}$ slices using a microtome and stained with haematoxylin (Vector Laboratories, USA) and eosin (Sigma-Aldrich, USA). The sliced tissues were examined by light microscopy. The heart injury score was determined in sections containing the right and left ventricles using a semi-quantitative scale from 0 to 4, as follows: $0=$ no injury, $1=$ isolated myocyte injury, $2=$ one focal area of injury, $3=$ two or more areas of injury, and $4=$ diffuse areas of damage compromising more than $50 \%$ of the myocardium.

\section{Statistics}

The differences between groups were analysed using an unpaired $t$-test using GraphPad Prism 5.01 (GraphPad Software, USA). Statistical significance was set to less than 0.05. The sample size was calculated with the "resource equation method". A sample size of 10 animals per group was calculated, as 
needed, to show a significant difference. The data are presented as the means \pm SD.

\section{Results}

Twenty rats were used for the experiments and allocated evenly into the two groups. The expression of ROS in blood from the propofol group was significantly lower than in the isoflurane group (54.32 $\pm 5.31 \%$ vs. $81.81 \pm 2.34 \%, p<0.05$ ) (Figure 1 ).

Cardiomyocyte apoptosis was significantly lower in the propofol group $(7.50 \pm 0.89 \%$ vs. $15.24 \pm$ $2.03 \%, p<0.05$ ) (Figure 2). Annexin-V was also lower in the propofol group (blood, $6.75 \pm 1.20 \%$ vs. $17.51 \pm$ $2.45 \%, p<0.05$; heart, $2.16 \pm 0.41 \%$ vs. $16.88 \pm 4.52 \%$, $p<0.05$ ) (Figure 2).
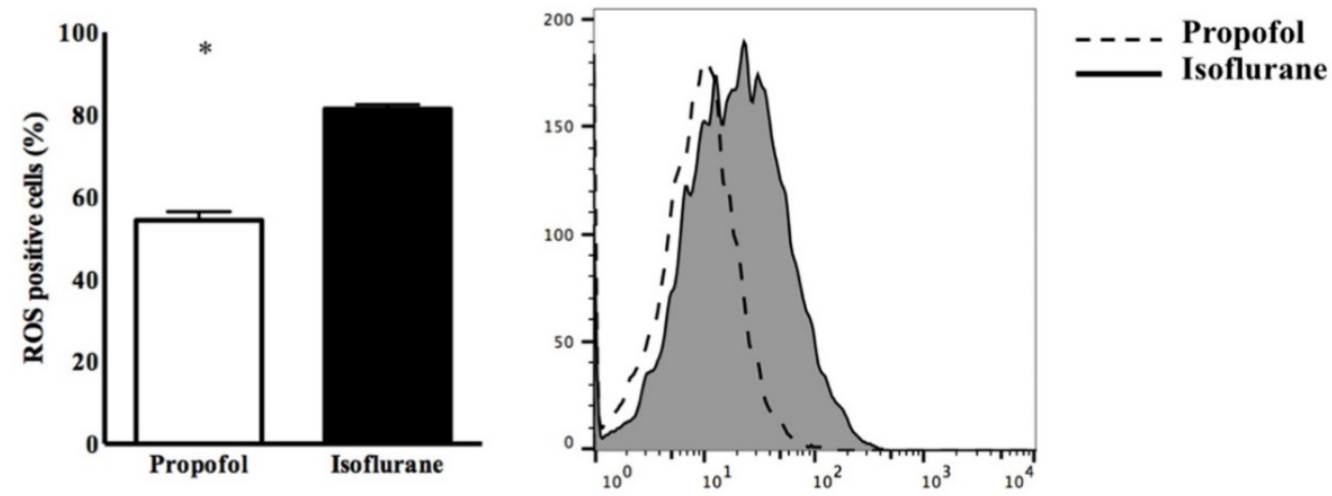

Figure 1. Expression of intracellular reactive oxygen species (ROS) in PBMC. ${ }^{*} p<0.05$ compared with Isoflurane group.
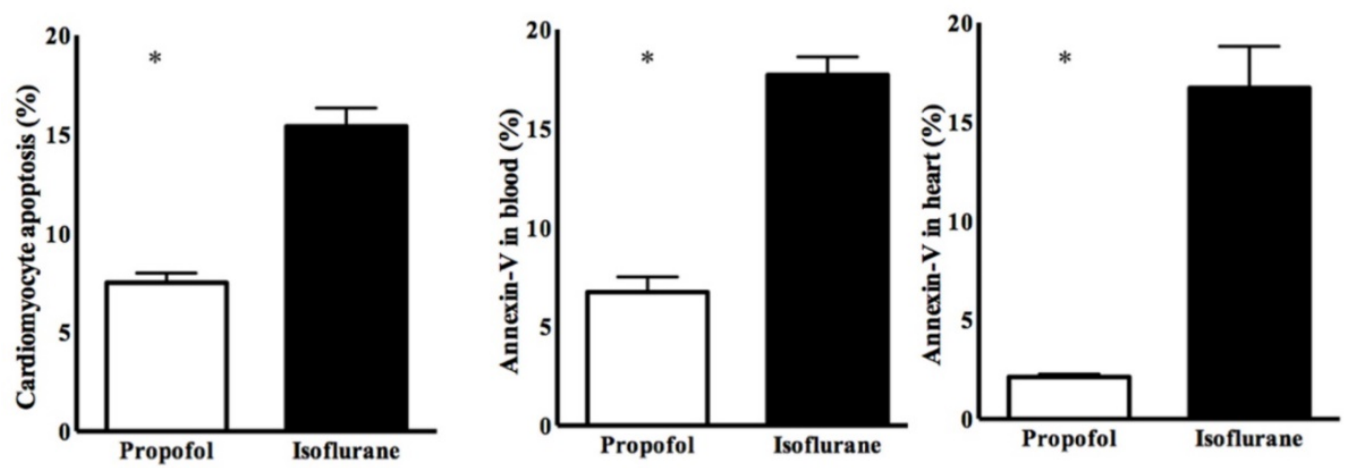

Figure 2. Cardiomyocyte apoptosis determined by annexin- $V$ staining. ${ }^{*} p<0.05$ compared with Isoflurane group.

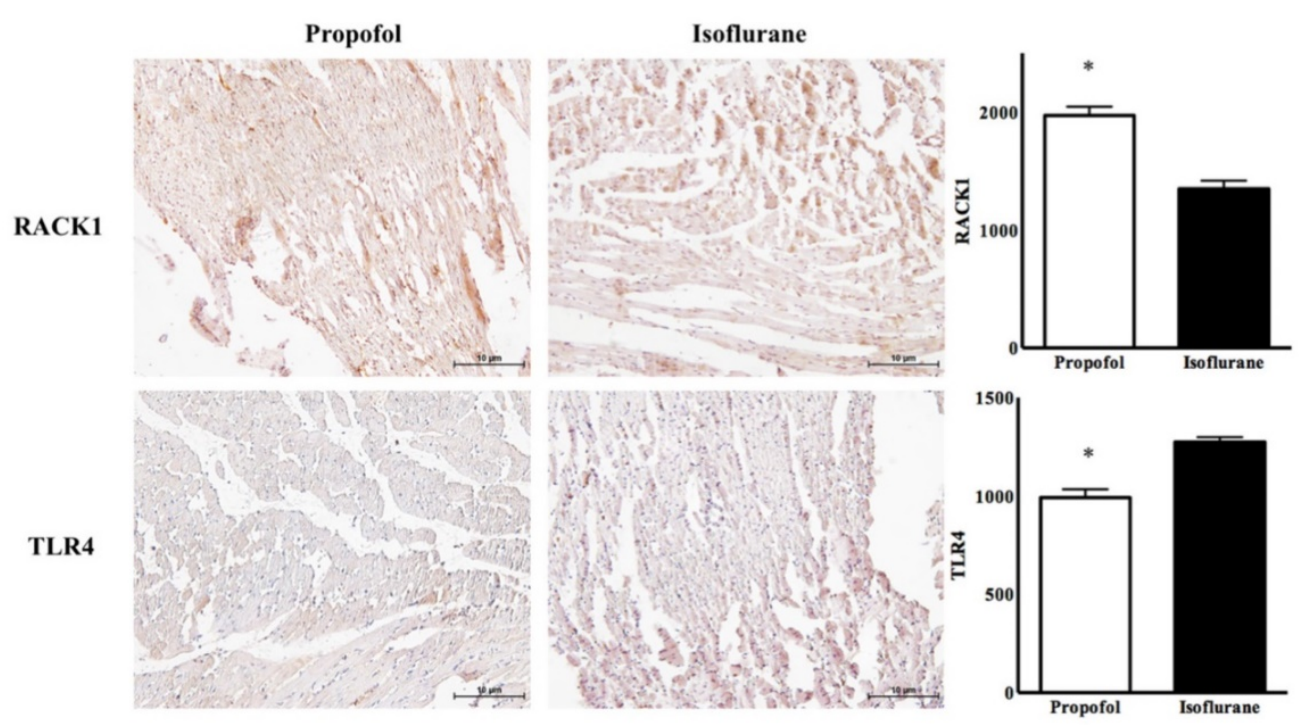

Figure 3. Immunohistochemistric findings for Receptor for activated C kinase 1 (RACK1) and toll-like receptor 4 (TLR4) in cardiomyocyte. ${ }^{*} p<0.05$ compared with Isoflurane group. 

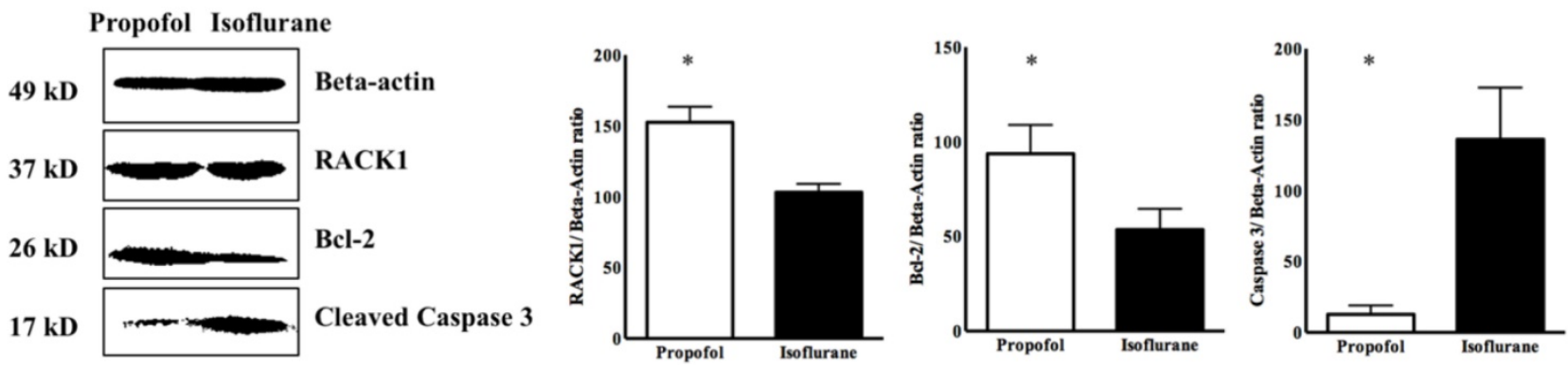

Figure 4. Western blot for Receptor for activated C kinase 1 (RACK1) ratio in cardiomyocyte. ${ }^{*} p<0.05$ compared with Isoflurane group.
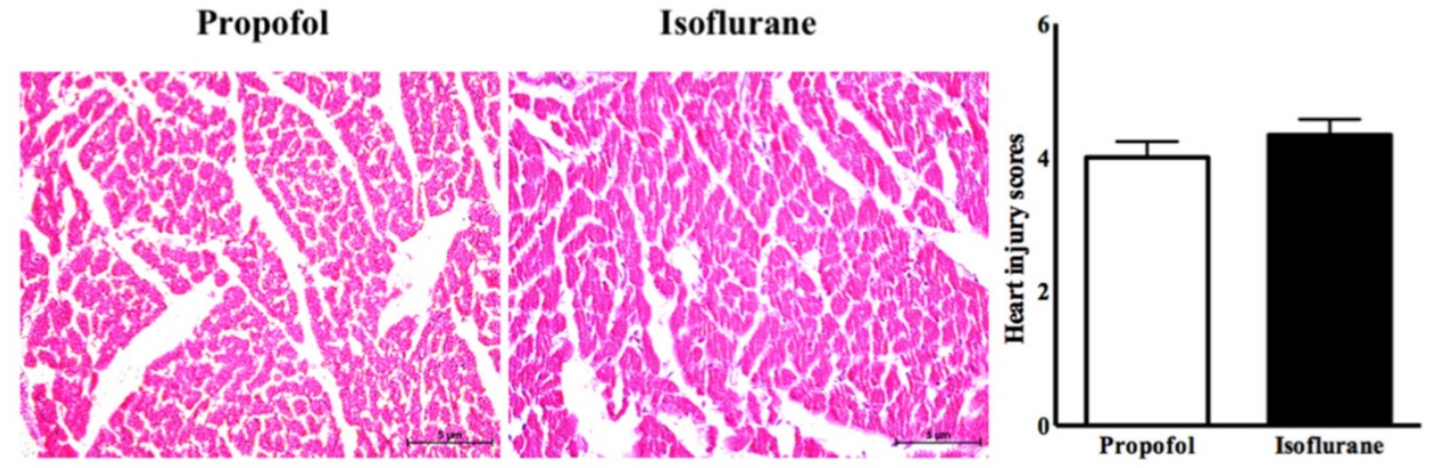

Figure 5. Histologic analysis of heart with heart injury score.

In the propofol group, the RACK1 expression was significantly higher and the TLR4 expression was lower, compared with the isoflurane group (RACK1, $1970.50 \pm 120.50$ vs. $1350.20 \pm 250.30, p<0.05$; TLR4, $980.50 \pm 110.75$ vs. $1275.50 \pm 75.35, p<0.05$ ) (Figure 3). Western blotting showed a similar pattern for the ratio of RACK1, Bcl-2, and cleaved caspase 3 (propofol vs. isoflurane, respectively: RACK1, $152.50 \pm$ 6.29 vs. $103.01 \pm 3.43, p<0.05$; $\mathrm{Bcl}-2,93.65 \pm 8.76$ vs. $53.63 \pm 6.29, p<0.05$; cleaved caspase $3,12.71 \pm 3.68$ vs. $30.60 \pm 21.18, p<0.05$ ) (Figure 4).

In the histopathological analysis, the two groups had similar heart injury scores $(4.00 \pm 0.23$ vs. $4.33 \pm$ $0.24, p=0.33$, propofol $v s$. isoflurane, respectively) (Figure 5).

\section{Discussion}

The propofol group showed significantly lower expression of ROS after myocardial IRI with significantly less cardiomyocyte apoptosis with lower annexin-V levels compared with the isoflurane group. The propofol group also had higher RACK1 expression and lower TLR4 expression. However, the heart injury scores in the two groups were similar.

The massive burst of ROS released during myocardial IRI results in cardiac injury and contractile dysfunction with increased infarct size [16, 17]. By contrast, the small releases of ROS before an ischemic event may have a beneficial effect on the heart at myocardial IRI [18]. In terms of ROS, propofol protects the heart against myocardial IRI via its ROS scavenging activity. It enhances the endogenous cardiac anti-oxidant capacity and ultimately attenuates IRI [19]. In comparison, inhalation anaesthetic agents provide protection against myocardial IRI by stimulating the release of small amounts of ROS, which triggers and enhances the production of endogenous anti-oxidant enzymes and activates mitochondrial $\mathrm{K}_{\mathrm{ATP}}$ channels, limiting myocardial infarction [20]. The significant lower expression of ROS in the propofol group in our study was reasonable.

The death of cardiomyocytes after myocardial IRI results from necrosis and apoptosis. ROS are involved in the cardiomyocyte apoptosis pathway [21]. Propofol has a similar chemical structure to the active nucleus of a-tocopherol, which has anti-oxidant effects. Jin et al. confirmed the dose-dependent protective effect of propofol in myocardial IRI, reducing cardiomyocyte apoptosis, compared with a control group [22]. Wu et al. demonstrated that isoflurane had a protective effect on cardiomyocytes exposed during myocardial IRI by reducing excess ROS production in culture with cardiomyocytes, compared with a control group [23]. The protective effect of isoflurane on cardiomyocytes during myocardial IRI is related to reducing pro-inflammatory cytokine production, eliminating 
oxidative stress, improving superoxide dismutase activity, and inhibiting cardiomyocyte apoptosis [24, 25]. In this study, the lower cardiomyocyte apoptosis in the propofol group was associated with the lower expression of ROS, compared with the isoflurane group.

Several studies have demonstrated that TLR4 has a role in myocardial IRI [26]. TLR4 activation increases ROS production in various forms of IRI [27]. Oyama et al. revealed that TLR4-deficient mice had smaller infarcts with less inflammation after myocardial IRI [28]. Therefore, the TLR4 pathway was the therapeutic target, achieving a reduction in ROS production, to protect against IRI [29-31]. Considering the association between TLR4 and ROS, RACK1 should control the TLR4 pathway and ROS production in myocardial IRI. Jia et al. showed that RACK1 protected against IRI by attenuating ROS production in $\mathrm{H} 9 \mathrm{C} 2$ cells, a rat cardiac myoblast cell line [32]. Therefore, the higher expression of RACK1 might inhibit the TLR4 pathway and reduce ROS production in myocardial IRI. In our study, the higher RACK1 expression in the propofol group was associated with the significantly lower expression of ROS and cardiomyocyte apoptosis with the significantly lower TLR4 expression.

Nevertheless, our result for isoflurane should be considered. First, the cardioprotective effect of isoflurane on myocardial IRI is initiated by the small amount of ROS released, not resulting in injury. Therefore, cardiomyocyte apoptosis would differ significantly between the two groups. Second, we could not assess the cardioprotective effect of isoflurane in myocardial IRI without a control group. Our results were a simple comparison between two groups. If rats without any anaesthetic agent were included, the results would be more concrete. However, ethical principles have to be considered. Third, the superiority of the cardioprotective effects of propofol and isoflurane could differ with the concentrations of anaesthetic agents, duration of the ischemic event, and other factors [33, 34].

Interestingly, the similar histological findings in the heart were remarkable. This meant that the significant differences in inflammation and apoptosis between the two groups were not sufficient to have a different effect on the injury. It also means that the myocardial function would be similar after myocardial IRI, although it was not evaluated in this study.

In conclusion, there were significant differences in inflammation and apoptosis, including the expression of RACK1 and TLR4, after myocardial IRI between the propofol and isoflurane groups. However, both groups had similar heart injury scores.
These findings indicate that propofol and isoflurane have similar effects on myocardial IRI, despite the different mechanisms involved.

\section{Acknowledgement}

This research was supported by Basic Science Research Program through the National Research Foundation of Korea (NRF) funded by the Ministry of Science, ICT and future Planning (Grant number: 2015R1A2A2A01006779, 2015).

This study was supported by the National Research Foundation of Korea (NRF) grant funded by the Korea government (NRF-2016R1A5A2012284).

This paper was written as part of Konkuk University's research support program for its faculty on sabbatical leave in 2017.

\section{Competing Interests}

The authors have declared that no competing interest exists.

\section{References}

1. Kalogeris T, Baines CP, Krenz M, Korthuis RJ. Cell biology of ischemia/reperfusion injury. Int Rev Cell Mol Biol. 2012; 298: 229-317.

2. Frangogiannis NG. The inflammatory response in myocardial injury, repair, and remodelling. Nat Rev Cardiol. 2014; 11: 255-65.

3. Sanderson TH, Reynolds CA, Kumar R, Przyklenk K, Huttemann M. Molecular mechanisms of ischemia-reperfusion injury in brain: pivotal role of the mitochondrial membrane potential in reactive oxygen species generation. Mol Neurobiol. 2013; 47: 9-23.

4. Boros P, Bromberg JS. New cellular and molecular immune pathways in ischemia/reperfusion injury. Am J Transplant. 2006; 6: 652-8.

5. Shibata R, Sato K, Pimentel DR, Takemura Y, Kihara S, Ohashi K, et al. Adiponectin protects against myocardial ischemia-reperfusion injury through AMPK- and COX-2-dependent mechanisms. Nat Med. 2005; 11: 1096-103.

6. Scarabelli T, Stephanou A, Rayment N, Pasini E, Comini L, Curello S, et al. Apoptosis of endothelial cells precedes myocyte cell apoptosis in ischemia/reperfusion injury. Circulation. 2001; 104: 253-6.

7. Qian L, Shi JH, Zhang C, Lu JW, Lu XN, Wu KP, et al. Downregulation of RACK1 is associated with cardiomyocyte apoptosis after myocardial ischemia/reperfusion injury in adult rats. In Vitro Cell Dev-An. 2016; 52: 305-13.

8. Cheng X, Hou J, Liu J, Sun X, Sheng Q, Han P, et al. Safety Evaluation of Sevoflurane as Anesthetic Agent in Mouse Model of Myocardial Ischemic Infarction. Cardiovasc Toxicol. 2017; 17: 150-6.

9. Yang C, Liu X, Yang F, Zhang W, Chen Z, Yan D, et al. Mitochondrial phosphatase PGAM5 regulates Keap1-mediated Bcl-xL degradation and controls cardiomyocyte apoptosis driven by myocardial ischemia/reperfusion injury. In Vitro Cell Dev Biol Anim. 2017; 53: 248-57.

10. Bright R, Mochly-Rosen D. The role of protein kinase $C$ in cerebral ischemic and reperfusion injury. Stroke. 2005; 36: 2781-90.

11. Adams DR, Ron D, Kiely PA. RACK1, A multifaceted scaffolding protein: Structure and function. Cell Commun Signal. 2011; 9: 22.

12. Al-Reefy S, Osman H, Jiang W, Mokbel K. Evidence for a pro-apoptotic function of RACK1 in human breast cancer. Oncogene. 2010; 29: 5651; author reply 2 .

13. Zarbock A, Eroglu A, Erturk E, Ince C, Westphal M. Ischemia-Reperfusion Injury and Anesthesia. Biomed Res Int. 2014.

14. Bae HB. Volatile anesthetics and ischemia-reperfusion injury. Korean J Anesthesiol. 2015; 68: 211-2

15. Frank JA, Gutierrez JA, Jones KD, Allen L, Dobbs L, Matthay MA. Low tidal volume reduces epithelial and endothelial injury in acid-injured rat lungs. Am J Respir Crit Care Med. 2002; 165: 242-9.

16. Zweier JL, Flaherty JT, Weisfeldt ML. Direct measurement of free radical generation following reperfusion of ischemic myocardium. Proc Natl Acad Sci US A. 1987: 84: 1404-7.

17. Bolli R, Patel BS, Jeroudi MO, Lai EK, McCay PB. Demonstration of free radical generation in "stunned" myocardium of intact dogs with the use of the spin trap alpha-phenyl N-tert-butyl nitrone. J Clin Invest. 1988; 82: 476-85.

18. Ambrosio G, Tritto I, Chiariello $M$. The role of oxygen free radicals in preconditioning. J Mol Cell Cardiol. 1995; 27: 1035-9. 
19. Xia Z, Godin DV, Ansley DM. Propofol enhances ischemic tolerance of middle-aged rat hearts: effects on $15-\mathrm{F}(2 \mathrm{t})$-isoprostane formation and tissue antioxidant capacity. Cardiovasc Res. 2003; 59: 113-21.

20. Tanaka K, Weihrauch D, Kehl F, Ludwig LM, LaDisa JF, Jr., Kersten JR, et al. Mechanism of preconditioning by isoflurane in rabbits: a direct role for reactive oxygen species. Anesthesiology. 2002; 97: 1485-90.

21. Maulik N, Yoshida T, Das DK. Oxidative stress developed during the reperfusion of ischemic myocardium induces apoptosis. Free Radic Biol Med. 1998; 24: 869-75.

22. Jin YC, Kim W, Ha YM, Shin IW, Sohn JT, Kim HJ, et al. Propofol limits rat myocardial ischemia and reperfusion injury with an associated reduction in apoptotic cell death in vivo. Vascul Pharmacol. 2009; 50: 71-7.

23. $\mathrm{Wu} \mathrm{W}$, Zhou $\mathrm{X}$, Liu $\mathrm{P}$, Fei $\mathrm{W}$, $\mathrm{Li} \mathrm{L}$, Yun $\mathrm{H}$. Isoflurane reduces hypoxia/reoxygenation-induced apoptosis and mitochondrial permeability transition in rat primary cultured cardiocytes. BMC Anesthesiol. 2014; 14: 17.

24. Liu M, Zhang H, Zhang Q, Huang C, Shi X. Syntaxin 1A mediates isoflurane but not hypoxia preconditioning-induced alleviation of hypoxia-reoxygenation injury in rat cardiomyocytes. Am J Transl Res. 2015; 7: 1883-95.

25. Liu J, Yang S, Zhang X, Liu G, Yue X. Isoflurane reduces oxygen-glucose deprivation-induced oxidative, inflammatory, and apoptotic responses in H9c2 cardiomyocytes. Am J Transl Res. 2016; 8: 2597-608.

26. Lee SM, Hutchinson M, Saint DA. The role of Toll-like receptor 4 (TLR4) in cardiac ischaemic-reperfusion injury, cardioprotection and preconditioning. Clin Exp Pharmacol Physiol. 2016; 43: 864-71.

27. Feng Y, Chao W. Toll-like receptors and myocardial inflammation. Int J Inflam. 2011; 2011: 170352

28. Oyama J, Blais C, Jr., Liu X, Pu M, Kobzik L, Kelly RA, et al. Reduced myocardial ischemia-reperfusion injury in toll-like receptor 4-deficient mice. Circulation. 2004; 109: 784-9.

29. Li YW, Zhang Y, Zhang L, Li X, Yu JB, Zhang HT, et al. Protective effect of tea polyphenols on renal ischemia/reperfusion injury via suppressing the activation of TLR4/NF-kappaB p65 signal pathway. Gene. 2014; 542: 46-51.

30. $\mathrm{Hu} \mathrm{N}$, Zhang Y. TLR4 knockout attenuated high fat diet-induced cardiac dysfunction via NF-kappaB/JNK-dependent activation of autophagy. Biochim Biophys Acta. 2017.

31. Arslan F, Keogh B, McGuirk P, Parker AE. TLR2 and TLR4 in ischemia reperfusion injury. Mediators Inflamm. 2010; 2010: 704202

32. Jia X, Zhang L, Mao X. S-propranolol protected H9C2 cells from ischemia/reperfusion-induced apoptosis via downregultion of RACK1 Gene. Int J Clin Exp Pathol. 2015; 8: 10335-44.

33. Yildirim V, Doganci S, Aydin A, Bolcal C, Demirkilic U, Cosar A. Cardioprotective effects of sevoflurane, isoflurane, and propofol in coronary surgery patients: a randomized controlled study. Heart Surg Forum. 2009; 12: E1-9.

34. Flier S, Post J, Concepcion AN, Kappen TH, Kalkman CJ, Buhre WF. Influence of propofol-opioid vs isoflurane-opioid anaesthesia on postoperative troponin release in patients undergoing coronary artery bypass grafting. Br J Anaesth. 2010; 105: 122-30. 